\title{
Initial assessment, level of care and outcome among children who were seen by emergency medical services: a prospective observational study
}

\author{
Carl Magnusson ${ }^{1 *}$ (D), Johan Herlitz ${ }^{1,2}$, Thomas Karlsson ${ }^{3}$ and Christer Axelsson ${ }^{1,2}$
}

\begin{abstract}
Background: The assessment of children in the Emergency Medical Service (EMS) is infrequent representing 5.4\% of the patients in an urban area in the western part of Sweden. In Sweden, patients are assessed on scene by an EMS nurse whom independently decides on interventions and level of care. To aid the EMS nurse in the assessment a triage instrument, Rapid Emergency Triage and Treatment System-paediatrics (RETTS-p) developed for Emergency Department (ED) purpose has been in use the last 5 years. The aim of this study was to evaluate the EMS nurse assessment, management, the utilisation of RETTS-p and patient outcome.
\end{abstract}

Methods: A prospective, observational study was performed on 651 children aged $<16$ years from January to December 2016. Statistical tests used in the study were Mann-Whitney U test, Fisher's exact test and Spearman's rank statistics.

Results: The dispatch centre indexed life-threatening priority in $69 \%$ of the missions but, of all children, only $6.1 \%$ were given a life threatening RETTS-p red colour by the EMS nurse. A total of $69.7 \%$ of the children were transported to the $\mathrm{ED}$ and, of these, 31.7\% were discharged without any interventions. Among the non-conveyed patients, 16 of 197 (8.1\%) visited the ED within $72 \mathrm{~h}$ but only two were hospitalised. Full triage, including five out of five vital signs measurements and an emergency severity index, was conducted in $37.6 \%$ of all children. A triage colour was not present in 146 children $(22.4 \%)$, of which the majority were non-conveyed. The overall 30 -day mortality rate was $0.8 \%(n=5)$ in children $0-15$ years.

Conclusions: Despite the incomplete use of all vital signs according to the RETTS-p, the EMS nurse assessment of children appears to be adapted to the clinical situation in most cases and the patients appear to be assessed to the appropriate level of care but indicating an over triage. It seems that the RETTS-p with full triage is used selectively in the pre-hospital assessment of children with a risk of death during the first 30 days of less than $1 \%$.

Keywords: Triage, Children, Pre-hospital assessment, Patient safety, EMS nurse, Level of care

\footnotetext{
* Correspondence: carl.magnusson@vgregion.se

${ }^{1}$ Department of Molecular and Clinical Medicine, Sahlgrenska Academy,

University of Gothenburg, Gothenburg, Sweden

Full list of author information is available at the end of the article
}

(c) The Author(s). 2018 Open Access This article is distributed under the terms of the Creative Commons Attribution 4.0 International License (http://creativecommons.org/licenses/by/4.0/), which permits unrestricted use, distribution, and reproduction in any medium, provided you give appropriate credit to the original author(s) and the source, provide a link to the Creative Commons license, and indicate if changes were made. The Creative Commons Public Domain Dedication waiver (http://creativecommons.org/publicdomain/zero/1.0/) applies to the data made available in this article, unless otherwise stated. 


\section{Background}

The Emergency Medical Services (EMS) have developed rapidly over the last decades with the ability to perform interventions on scene which requires competence and clinical judgment to adequately assess the patient's condition. In Sweden all ambulances are manned by a registered nurse, often with specialist training. The EMS nurses have been given the responsibility to assess patients at the scene and independently decide on treatment and level of care. To aid the EMS nurse in the assessment a triage protocol is being used. The Rapid Emergency Triage and Treatment System-paediatrics (RETTS-p) was initially developed for triaging within the paediatric Emergency Department (pED), and the majority of the pEDs at university hospitals use the RETTS-p. The pED in the western part of Sweden have approximately 50,000 visits each year with a hospital admission rate of $14 \%$ and have been using the RETTS-p since 2010. The EMS, organised under the university hospital, implemented the RETTS-p during 2014 to start the triage process at an early stage in the chain of care. The RETTS-p is a five-level scale including vital signs (VS) in each patient assessment. Five-level triage scales have shown a higher accuracy identifying critical ill patients compared to three-level triage scales $[1,2]$. The Canadian paediatric triage and acuity scale (pedCTAS), Manchester triage system (MTS), Emergency severity index (ESI), and the Australasian triage scale (ATS) are all based on expert opinion such as the RETTS-p and are common in the pED [3]. They have shown moderate to good reliability and validity [4-11]. The ESI have in several studies in different locations shown an association of $80-100 \%$ predicting hospital admission in the highest triage levels $[9,12-14]$. Validity studies are lacking for the RETTS-p but previous studies have shown a good to a very good reliability between nurses in the $\mathrm{pED}[15,16]$. The accuracy of triage in the EMS has been extensively studied in the assessment of trauma patients, indicating difficulties in the assessment of the patient with both over-triage and under-triage [17-20]. A five-level triage tool in the EMS might be favourable for detecting a severely ill patient [21]. The use of triage in the EMS to assess paediatric patients was proposed 25 years ago [22]. However, implementing more complex triage systems for adult patients has shown only moderate agreement between the EMS assessment and ED nurses [23, 24]. Furthermore, the appropriateness of the EMS nurse assessment utilising an pED triage system is unknown. Thus, there is a knowledge gap whether it is feasible to apply it in the pre-hospital setting in order to triage the patient to the appropriate triage level in the pED. The aim of this study was therefor to evaluate the initial priority given by the dispatcher,the EMS nurse assessment, management, utilisation of the RETTS-p and finally patient outcome in an unselected population of children under the age of 16 .

\section{Methods \\ Design}

The present study was a prospective observational study of paediatric emergency patients who were assessed by an EMS nurse.

\section{RETTS-p}

The RETTS-p is made up of two parts, vital signs (VS) and emergency signs and symptoms (ESS). The level of severity is based on the highest colour of ESS or VS that becomes the final triage level. Red is stated as life threatening, orange is potentially life threatening and both levels are, from a time perspective, defined as emergency care directly. Yellow and green are defined as no individual medical risk if put on wait for assessment by a physician. Five VS, respiratory rate, oxygen saturation, pulse frequency, body temperature and level of consciousness, are recorded and adjusted to age intervals, including pulse correction for patients presenting with fever. An upset child can affect the pulse rate, and this may prevent the child from showing a reliable VS [25]. The level of consciousness is defined by the RETTS-p as "alert" (Green), "tired/crummy" (Yellow), GCS 11-13 (Orange) and GCS $\leq 10$ or ongoing seizures (Red). There are a total of 40 ESS cards with the most common complaints. The RETTS-p is not constructed as an instrument to decide whether the patient qualifies for emergency care nor to reject a patient emergency care in a pre-hospital context. However, the lowest triage level, blue category, means that, after assessment, the patient could be referred to other levels of care with no medical risk. Within the EMS organisation included in this study, only levels green to red are currently being used.

\section{Settings}

The study was conducted within an EMS organisation operating in an urban area in the western part of Sweden. The EMS organisation covers an area of $900 \mathrm{~km}^{2}$ with a population of 660,000 inhabitants and with predominantly short transportation times. During the year of 2016, the EMS carried out more than 80,000 ambulance missions (priority 1 to 3 ) and, of these, 58,575 assignments involve an initial patient assessment defined as a primary mission (Fig. 1). Approximately $3150(5.4 \%)$ of these missions involve children aged < 16 years. The Emergency medical dispatch centre prioritise the patient with help from a Dispatch Medical Index (DMI). The DMI originally developed in the US has been adapted and used in Sweden since 1995 [26]. Based on the DMI a priority is given to the mission and an ambulance is allocated. Priority 1 is defined as life threatening 


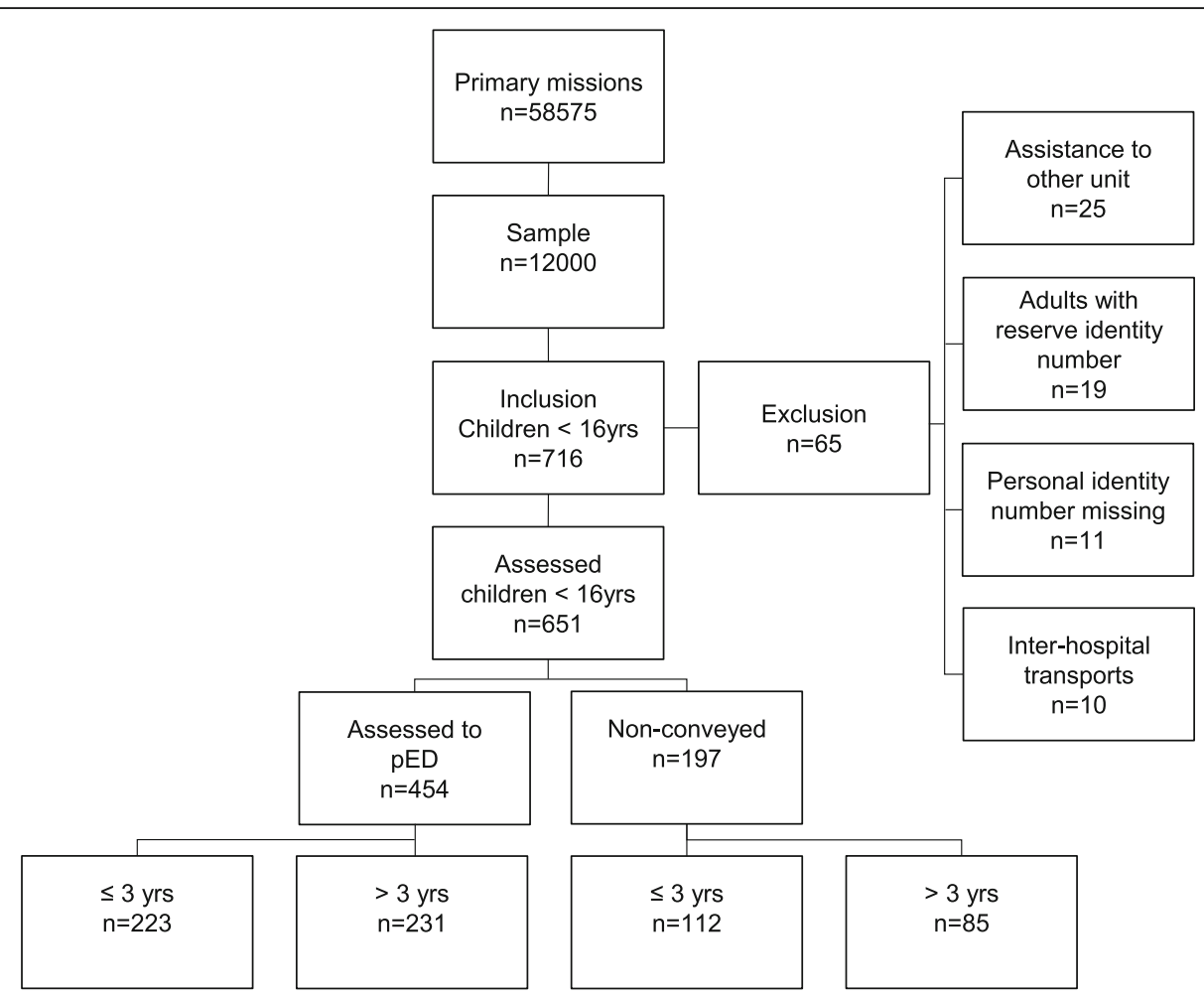

Fig. 1 Flow chart of the studied patients, the distribution of patient assesment and median age

to which the EMS responds with blue lights and sirens, priority 2 is urgent but not life threatening and priority 3 is assignments where the waiting time would not affect the medical condition. All the ambulances within the organisation are advanced life support (ALS) units and each ambulance is manned by at least one registered nurse responsible for patient assessment and the administration of drugs according to Swedish regulations. The EMS organisation aims to increase the number of specialist nurses trained in pre-hospital care, which comprises of a oneyear postgraduate education in pre-hospital care. Fifty per cent of the staff within the EMS organisation are specialist nurses with this training.

\section{Materials}

The patients in this study were included consecutively from a larger sample of the first 1000 assignments each month (January-December) in $2016(n=12,000)$. The inclusion criterion was an EMS assignment where a patient assessment took place at the scene. The exclusion criteria were as follows: 1) patients $>16$ years, 2) assignments with no patient contact, 3) assignments assisting other EMS units and 4) inter-hospital transportation. A total of 716 patients under the age of 16 were initially identified from the sample. After a manual review, 65 patients were excluded as they did not fulfil the criteria and a total of 651 patients were finally included in the study (Fig. 1). Data were collected from EMS records and hospital records. Scanned patient records in which medications are prescribed in the pED were also reviewed for each patient.

\section{Statistical analysis}

In the tables, results are presented as the number (percentage) or median (25th, 75th percentile). For two-group comparisons, the Mann-Whitney U test was used, while Fisher's exact test was used for continuous/ordered and dichotomous/categorical variables respectively. To test for associations with age, Spearman's rank statistics were used for continuous/ordered variables and the Mann-Whitney $\mathrm{U}$ test was used for dichotomous/categorical variables. The actual age (in years) was used for $p$-value calculations (Tables 1,2,3), although in Table 2 data are presented for patients dichotomised by the median ( 3 years). All tests are two sided and, due to the number of tests performed, $p$-values below 0.01 were regarded as statistically significant. Data processing and statistical analysis were performed using SPSS version 22.

\section{Results}

\section{Children allocated to pED or non-conveyed}

The median age for all children was 3 years. Among children $<16$ years of age, $30.3 \%$ were non-conveyed after assessment, with a referral to primary care or with 
Table 1 Children allocated to pED or non-conveyed

\begin{tabular}{|c|c|c|c|}
\hline & Assessed to $\mathrm{pED} n=454$ & Non-conveyed $n=197$ & $P$ \\
\hline \multicolumn{4}{|l|}{ Age - year (25th, 75th percentile) } \\
\hline Median & $4(1,11)$ & $2(1,7)$ & 0.002 \\
\hline \multicolumn{4}{|l|}{ Gender - n(\%) } \\
\hline Female & $194(42.7)$ & $86(43.7)$ & 0.863 \\
\hline Dispatcher priority - n(\%) & & & $<0.001$ \\
\hline Priority 1 & $336(74.0)$ & $113(57.3)$ & \\
\hline Priority 2 & $113(24.9)$ & $75(38.1)$ & \\
\hline Priority 3 & $5(1.1)$ & $9(4.6)$ & \\
\hline \multicolumn{4}{|l|}{ Dispatch classification index ${ }^{a}-n(\%)$} \\
\hline Respiratory distress & 89 (19.6) & $51(25.9)$ & 0.078 \\
\hline Fever & $55(12.1)$ & $34(17.3)$ & 0.083 \\
\hline Convulsions ongoing/terminated & $63(13.9)$ & $11(5.6)$ & 0.002 \\
\hline Major trauma high energy/ large bleeding/ headinjury & $54(11.9)$ & $14(7.1)$ & 0.071 \\
\hline Minor trauma extremity/ wound/ fracture & $46(10.1)$ & $21(10.7)$ & 0.888 \\
\hline Time of day - n(\%) & & & 0.034 \\
\hline 08:00-16:00 & $163(35.9)$ & $58(29.5)$ & \\
\hline $16: 00-24: 00$ & $221(48.7)$ & $96(48.7)$ & \\
\hline 24:00-08:00 & $70(15.4)$ & $43(21.8)$ & \\
\hline \multicolumn{4}{|l|}{ Medical history ${ }^{\mathrm{b}}$ - n(\%) } \\
\hline No medical history & $297(65.4)$ & $132(67.0)$ & 0.720 \\
\hline Asthma, common cold with asthma & $27(5.9)$ & $15(7.6)$ & 0.487 \\
\hline Congenital disability & $30(6.6)$ & $12(6.1)$ & 0.864 \\
\hline Febrile non-epileptical convulsions, absences & $24(5.3)$ & $7(3.6)$ & 0.425 \\
\hline Allergies & $18(4.0)$ & $8(4.1)$ & 0.999 \\
\hline \multicolumn{4}{|l|}{ Vital signs - median (25th, 75th percentile) } \\
\hline Respiratory rate/min $(141,98)^{c}$ & $22(18,30)$ & $22(20,29)$ & 0.800 \\
\hline Saturation \% $(71,72)$ & $98(97,100)$ & $99(98,100)$ & 0.003 \\
\hline Pulse rate/min $(79,74)$ & $117(90,140)$ & $115(95,140)$ & 0.858 \\
\hline Temperature ${ }^{\circ} \mathrm{C}(133,86)$ & $37.3(36.7,38.4)$ & $37.2(36.7,38.1)$ & 0.880 \\
\hline Level of consciousness according to RETTS-p - n(\%) $(81,26)$ & & & $<0.001$ \\
\hline Alert & $276(74.0)$ & $162(94.7)$ & \\
\hline Tired/ Crummy & $54(14.5)$ & $8(4.7)$ & \\
\hline GCS 11-13 & $30(8.0)$ & $1(0.6)$ & \\
\hline GCS $\leq 10 /$ Ongoing seizure & $13(3.5)$ & 0 & \\
\hline EMS nurse triage level according to RETTS-p - n(\%) $(64,82)^{c}$ & & & $<0.001$ \\
\hline Red & $40(10.3)$ & $0(0.0)$ & \\
\hline Orange & $109(27.9)$ & $4(3.4)$ & \\
\hline Yellow & $179(45.9)$ & $34(29.6)$ & \\
\hline Green & $62(15.9)$ & $77(67.0)$ & \\
\hline \multicolumn{4}{|l|}{ Assessed condition according to RETTS- $p^{d}-n(\%)(64,83)^{c}$} \\
\hline Trauma (head, thorax, extremity, burn) & $100(25.6)$ & $17(14.9)$ & $<0.001$ \\
\hline Respiratory difficulty (dyspnoea, Shortness of breath) & $58(14.9)$ & $28(24.6)$ & 0.023 \\
\hline Convulsion & $76(19.5)$ & $5(4.4)$ & $<0.001$ \\
\hline Fever & $26(6.7)$ & $20(17.5)$ & 0.001 \\
\hline
\end{tabular}


Table 1 Children allocated to pED or non-conveyed (Continued)

\begin{tabular}{|c|c|c|c|}
\hline & Assessed to $p E D n=454$ & Non-conveyed $n=197$ & $P$ \\
\hline Abdominal pain & $30(7.7)$ & $10(8.8)$ & 0.696 \\
\hline \multicolumn{4}{|l|}{ Drug administration - $n(\%)^{e}$} \\
\hline EMS nurse drug administration & $204(44.9)$ & $26(13.2)$ & $<0.001$ \\
\hline Antipyretics & $74(16.3)$ & $11(5.6)$ & $<0.001$ \\
\hline Local anesthetics & $43(9.5)$ & 0 & $<0.001$ \\
\hline Oxygen & $29(6.4)$ & $1(0.5)$ & $<0.001$ \\
\hline Analgetics & $29(6.4)$ & 0 & $<0.001$ \\
\hline Steroids & $25(5.5)$ & $8(4.1)$ & 0.561 \\
\hline \multicolumn{4}{|l|}{ Mortality - n(\%) } \\
\hline$<30$ days & $5(1.1)$ & 0 & 0.330 \\
\hline
\end{tabular}

${ }^{a}$ Dispatch index can consist of one or two indexes

${ }^{\mathrm{b}}$ The most common medical history, a patient can have more than one condition

'Missing vital signs, triage level and assessed condition for children assessed to pED and non-conveyed respectively

${ }^{\mathrm{d}}$ The most common assessed conditions according to RETTS-p

'The most common administered drugs, a patient could have been administered more than one

self-care advice. These non-conveyed children were significantly younger than those transported to the pED (Table 1). There was a significant association between a higher priority level assessed by dispatch and the patient being transported to the $\mathrm{pED}$. Thus, children assessed as being in need of pED care by the EMS nurse were given a significantly higher priority by dispatch. However, of the assignments with priority 1 from dispatch, only $7.8 \%$ were assessed as life threatening (RETTS-p red) by the EMS nurse and, in the total study population, only $6.1 \%$ of the patients were given a red triage level (Table 1). Respiratory distress was the most common DMI overall. The DMI for convulsions was significantly more common in the pED care group compared with those non-conveyed. There was no significant difference in EMS allocation regarding time of day between the two groups, but an overall decrease in patient contact during the night was observed. The majority of the children had no previous medical history, but, among those with a medical history, the most frequent previous conditions were asthma/common cold with asthma and congenital disability. Children assessed to the pED had a significantly greater reduction in oxygen saturation and a more affected level of consciousness than children who were not conveyed. In overall terms, a triage colour was missing in $22.4 \%$ of the assessments and the proportion was higher for children assessed to stay at the scene compared with patients transported to the pED. Patients assessed to pED care were associated with a significantly higher triage level. Trauma was the most common condition according to the RETTS-P assessment and, together with convulsions, it was significantly more common in those transported to the $\mathrm{pED}$, whereas fever was more common in the non-conveyance group. Significantly more children in the $\mathrm{pED}$ group received medical treatment by the EMS nurse. Children who died within 30 days $(n=5)$ were all initially transported to hospital (Table 1).

\section{Renewed contact within $72 \mathrm{~h}$}

Of the non-conveyed patients ( $n=197), 16(8.1 \%)$ visited the pED within $72 \mathrm{~h}$. Seven of these patients were transported by ambulance, one in a single manned technician patient transport and the remaining eight by their own transport. Three of the 16 patients were assessed, treated and discharged by a pED nurse, one patient was left before evaluation and 12 children were assessed by a physician. Of these 12, two were admitted to inpatient care, seven were discharged with an intervention/prescription and three patients were discharged without any intervention. One patient with a final diagnosis of anaphylaxis who initially received epinephrine, administered by one of the parents, was observed in the pED and was given additional treatment.

\section{Patient assessment and association with age}

There was a significant inverse association between age and dispatch priority. Respiratory distress and fever were the most common DMI among younger children and they were significantly associated with a lower age. For older children, minor trauma was the most common condition assessed by dispatch and it was significantly associated with a higher age (Table 2). There was no significant difference in age regarding time of day. Increased respiratory rate, decreased oxygen saturation and increased pulse frequency and body temperature were all significantly associated with a lower age, as was a lower level of consciousness (Table 2). The RETTS-P assessment of respiratory difficulty and convulsions was significantly associated with a lower age, whereas trauma 
Table 2 Patient assessment and association with age

\begin{tabular}{|c|c|c|c|}
\hline & Age $\leq 3$ years $n=335$ & Age $>3$ years $n=316$ & $P$ \\
\hline \multicolumn{4}{|l|}{ Gender - n(\%) } \\
\hline Female & $133(39.7)$ & $147(46.5)$ & 0.030 \\
\hline Dispatcher priority - n(\%) & & & $<0.001$ \\
\hline Priority 1 & $253(75.5)$ & $196(62.0)$ & \\
\hline Priority 2 & $75(22.4)$ & $113(35.8)$ & \\
\hline Priority 3 & $7(2.1)$ & $7(2.2)$ & \\
\hline \multicolumn{4}{|l|}{ Dispatch classification index ${ }^{a}-n(\%)$} \\
\hline Respiratory distress & $102(30.4)$ & $38(12.0)$ & $<0.001$ \\
\hline Fever & $64(19.1)$ & $25(7.9)$ & $<0.001$ \\
\hline Convulsions ongoing/ terminated & 49 (14.6) & $25(7.9)$ & 0.146 \\
\hline Major trauma high energy/ large bleeding/ headinjury & $32(9.6)$ & $36(11.4)$ & 0.243 \\
\hline Minor trauma exremity/ wound/ fracture & $19(5.7)$ & $48(15.2)$ & $<0.001$ \\
\hline Time of day - n(\%) & & & 0.015 \\
\hline 08:00-16:00 & $103(30.7)$ & $118(37.3)$ & \\
\hline 16:00-24:00 & $166(49.6)$ & $151(47.8)$ & \\
\hline 24:00-08:00 & $66(19.7)$ & $47(14.9)$ & \\
\hline \multicolumn{4}{|l|}{ Medical history ${ }^{\mathrm{b}}$ - n(\%) } \\
\hline No medical history & $230(68.7)$ & $199(63.0)$ & 0.015 \\
\hline Common cold with asthma/ asthma & $25(7.5)$ & $17(5.4)$ & 0.954 \\
\hline Congenital disability & $15(4.5)$ & $27(8.5)$ & 0.159 \\
\hline Febrile non-epileptical convulsions, absences & $24(7.2)$ & $7(2.2)$ & 0.168 \\
\hline Allergies & $11(3.3)$ & $15(4.7)$ & 0.178 \\
\hline \multicolumn{4}{|l|}{ Primary vital signs - median (25th, 75th percentile) } \\
\hline Respiratory rate/min $(164,75)^{c}$ & $30(24,40)$ & $20(16,22)$ & $<0.001$ \\
\hline Saturation \% $(103,40)$ & $98(97,100)$ & $99(98,100)$ & 0.001 \\
\hline Pulse rate/min $(113,40)$ & $140(120,160)$ & $98(83,117)$ & $<0.001$ \\
\hline Temperature ${ }^{\circ} \mathrm{C}(126,93)$ & $37.5(36.7,38.8)$ & $37.1(36.7,37.8)$ & $<0.001$ \\
\hline Level of consciousness according to RETTS-p - n(\%) $(58,49)$ & & & $<0.001$ \\
\hline Alert & $204(73.6)$ & $234(87.7)$ & \\
\hline Tired/ Crummy & $47(17.0)$ & $15(5.6)$ & \\
\hline GCS 11-13 & $19(6.9)$ & $12(4.5)$ & \\
\hline GCS $\leq 10 /$ Ongoing seizure & $7(2.5)$ & $6(2.2)$ & \\
\hline EMS nurse triage level according to RETTS-p - $n(\%)(99,47)^{c}$ & & & 0.134 \\
\hline Red & $19(8.1)$ & $21(7.8)$ & \\
\hline Orange & $52(22.0)$ & $61(22.7)$ & \\
\hline Yellow & $93(39.4)$ & $120(44.6)$ & \\
\hline Green & $72(30.5)$ & $67(24.9)$ & \\
\hline \multicolumn{4}{|l|}{ Assessed condition according to RETTS- $p^{d}-n(\%)(100,47)^{c}$} \\
\hline Trauma (head, thorax, extremity, burn) & $35(14.9)$ & $82(30.5)$ & $<0.001$ \\
\hline Respiratory difficulty (dyspnoea, shortness of breath) & $64(27.2)$ & $22(8.2)$ & $<0.001$ \\
\hline Convulsions & $55(23.4)$ & $26(9.7)$ & 0.002 \\
\hline Fever & $29(12.4)$ & $17(6.3)$ & 0.023 \\
\hline Abdominal pain & $5(2.1)$ & 35 (13.0) & $<0.001$ \\
\hline
\end{tabular}


Table 2 Patient assessment and association with age (Continued)

\begin{tabular}{|c|c|c|c|}
\hline & Age $\leq 3$ years $n=335$ & Age $>3$ years $n=316$ & $P$ \\
\hline \multicolumn{4}{|l|}{ EMS nurse Assessment - $\mathrm{n}(\%)$} \\
\hline Assessed to $\mathrm{pED}$ & $223(66.6)$ & $231(73.1)$ & 0.002 \\
\hline Renewed contact within $72 \mathrm{~h}^{\mathrm{e}}$ & $10(8.9)$ & $6(7.1)$ & 0.511 \\
\hline \multicolumn{4}{|l|}{ Drug administration - $\mathrm{n}(\%)^{\mathrm{f}}$} \\
\hline EMS nurse administration & $114(34.0)$ & $116(36.7)$ & 0.085 \\
\hline Antipyretics & $42(12.5)$ & $43(13.6)$ & 0.198 \\
\hline Local anesthetics & $18(5.4)$ & $25(7.9)$ & 0.092 \\
\hline Oxygen & $18(5.4)$ & $12(3.8)$ & 0.292 \\
\hline Analgesics & $3(0.9)$ & $26(8.2)$ & $<0.001$ \\
\hline Steroids & $16(4.8)$ & $17(5.4)$ & 0.441 \\
\hline \multicolumn{4}{|l|}{ Mortality - n(\%) } \\
\hline$<30$ days & $3(0.9)$ & $2(0.6)$ & 0.404 \\
\hline
\end{tabular}

${ }^{a}$ Dispatch index can consist of one or two indexes

${ }^{\mathrm{b}}$ The most common medical history, a patient can have more than one condition

${ }^{c}$ Missing vital signs, triage level and assessed condition for $\leq 3$ year and over 3 years respectively

${ }^{\mathrm{d}}$ The most common assessed conditions according to RETTS-p

ePatients initially non-conveyed that visited the pED within 72 hours

${ }^{\mathrm{f}}$ The most common administered drugs, a patient could have been administered more than one

and abdominal pain were associated with increasing age (Table 2). The most frequently administered drug was acetaminophen. The administration of analgesics (morphine, fentanyl and esketamine) was significantly associated with a higher age (Table 2).

\section{Adherence to the RETTS-p}

Of all the children assessed by the EMS nurse, 406 (62.4\%) did not receive a full-triage (5 VS + ESS) and, of these children, 146 did not receive any triage colour at all (Table 3). Children with a limited triage were significantly younger and were non-conveyed to a greater extent. The most common missing VS was respiratory rate (Table 1), which was also more frequently missing among children who stayed at the scene compared with transported children (49.7 and $31.1 \%$ respectively, $p<$ $0.001)$. There was no significant difference between the full triage and limited triage groups regarding admission to inpatient care or interventions in the pED (Table 3). A total of $412(90.7 \%)$ patients transported to the $\mathrm{pED}$ received a diagnosis according to the ICD-10. Among all these patients, the most frequent diagnosis groups were "injury, poisoning and certain other consequences of external causes" and these diagnosis groups were significantly more common among children who had a limited triage compared with those with a full triage (Table 3).

\section{Discussion}

Assignments involving children in pre-hospital emergency care are infrequent when related to the total number of ambulance missions in a national (Sweden) and an international perspective [27-30]. The use of a structured triage system supporting the EMS nurse in the assessment of children may therefore be advocated to assure a systematic assessment. However, one third of the patients in the study stayed at the scene, indicating low resource utilisation. These data suggest that management by other levels of care may be a feasible alternative in many cases and this has also previously been reported [31-34]. It is also known that dispatch priorities diverge from EMS assessments at the scene, resulting in dispatch over-triage [26,35]. This was also found in this study. However, there may be several reasons for the high dispatch priority. The operator at the dispatch centre has limited possibilities to assess the child over the phone, is restricted to the DMI and should decide on the priority within seconds according to dispatch regulations. The patient's condition is also a dynamic process where for example convulsions may have terminated at the arrival of the EMS. The available ambulance units may also play a role, where units with lower priority assignments can be cancelled and assigned to priority 1. Hence, if assigned to a lower priority, the time frame upon arrival may be uncertain for an ambulance to arrive at the scene. Furthermore, the EMS nurse at the scene administered drugs in $35 \%$ of patients for example inhalation drugs for acute obstructive laryngitis and the downgrade in priority on scene may also be explained by the fact that the intervention on scene lowered the triage level.

The diversity in the assessed severity of the condition between dispatch centre and the EMS indicates that other units, such as a single-responder unit, manned by one specialist trained and experienced nurse, may be a 
Table 3 Adherence to the RETTS-p

\begin{tabular}{|c|c|c|c|}
\hline & Full triage $n=245$ & Non-full triage $n=406$ & $P$ \\
\hline \multicolumn{4}{|l|}{ Age - year (25th, 75th percentile) } \\
\hline Median & $6(2,12)$ & $2(1,7)$ & $<0.001$ \\
\hline \multicolumn{4}{|l|}{ Gender - n(\%) } \\
\hline Female & $109(44.5)$ & $171(42.1)$ & 0.568 \\
\hline Dispatcher priority - $\mathrm{n}(\%)$ & & & 0.862 \\
\hline Priority 1 & $170(69.4)$ & $279(68.7)$ & \\
\hline Priority 2 & $70(28.6)$ & $118(29.1)$ & \\
\hline Priority 3 & $5(2.0)$ & $9(2.2)$ & \\
\hline \multicolumn{4}{|l|}{ EMS nurse assessment - $n(\%)$} \\
\hline Non-conveyed & $55(22.4)$ & $142(35.0)$ & 0.001 \\
\hline Renewed contact within $72 \mathrm{~h}^{\mathrm{a}}$ & $5(9.1)$ & $11(7.7)$ & 0.795 \\
\hline EMS nurse triage level according to RETTS-p - $n(\%)(0,146)^{b}$ & & & 0.607 \\
\hline Red & $15(6.1)$ & $25(6.2)$ & \\
\hline Orange & $56(22.9)$ & $57(14.0)$ & \\
\hline Yellow & $116(47.3)$ & $97(23.9)$ & \\
\hline Green & $58(23.7)$ & $81(20.0)$ & \\
\hline \multicolumn{4}{|l|}{ Drug administration - n(\%) } \\
\hline EMS nurse administration & $101(41.2)$ & $129(31.8)$ & 0.015 \\
\hline pED administration ${ }^{c}$ & $34(17.9)$ & $39(14.8)$ & 0.372 \\
\hline EMS nurse and pED administration ${ }^{d}$ & $41(21.6)$ & $61(23.1)$ & 0.701 \\
\hline \multicolumn{4}{|l|}{ Management pED - n(\%) } \\
\hline No intervention $\Rightarrow$ discharged & $53(27.9)$ & $91(34.5)$ & 0.153 \\
\hline Intervention prescription/min. surgery/x-ray $\Rightarrow$ discharged & $85(44.7)$ & $102(38.6)$ & 0.209 \\
\hline Admission to in-patient care & $52(27.4)$ & $71(26.9)$ & 0.915 \\
\hline \multicolumn{4}{|l|}{ Days of in-patient care - $\mathrm{n}$} \\
\hline Mean (SD) & $3.3(6.1)$ & $3.4(4.7)$ & 0.095 \\
\hline Median (25th,75th percentile) & $2(1,3)$ & $2(1,4)$ & 0.674 \\
\hline \multicolumn{4}{|l|}{ ICD-10 Codes - n(\%) $(69,170)^{\mathrm{b}}$} \\
\hline$(S, T)$ Injury, poisoning and certain other consequnces of external causes & $41(23.4)$ & $85(35.9)$ & 0.007 \\
\hline Fractures & $8(19.5)$ & $14(16.5)$ & \\
\hline Superficial injuries & $2(4.9)$ & $16(18.8)$ & \\
\hline (R) Symptoms, signs and abnormal clinical and laboratory findings & $53(30.3)$ & $54(22.8)$ & 0.090 \\
\hline Convulsions, not elsewhere classified & $25(47.2)$ & $35(64.8)$ & \\
\hline Unspecific abdominal pain & $10(18.9)$ & $3(5.5)$ & \\
\hline (J) Diseases of the respiratory system & $23(13.1)$ & $33(13.9)$ & 0.885 \\
\hline Acute obstructive laryngitis & $6(26.1)$ & $11(33.3)$ & \\
\hline Acute upper respiratory infections & $5(21.7)$ & $10(30.3)$ & \\
\hline$(A, B)$ Certain infectious and parasitic diseases & $16(9.1)$ & $22(9.3)$ & 0.999 \\
\hline Viralinfection of unspecified site & $7(43.8)$ & $15(68.2)$ & \\
\hline Infectious gastroenteritis and colitis, unspecified & $4(25.0)$ & $6(27.3)$ & \\
\hline \multicolumn{4}{|l|}{ Mortality - n (\%) } \\
\hline$<30$ days & $1(0.4)$ & $4(0.9)$ & 0.655 \\
\hline
\end{tabular}

${ }^{\text {aPatients initially non-conveyed }}$

${ }^{\mathrm{b}}$ Missing triage level and diagnose code for full triage and non-full triage

${ }^{\mathrm{C}}$ Administration of drugs in the pED identical to drugs in the ambulance and not administrated in the ambulance

${ }^{d}$ Administration of drugs in the ambulance and follow up dose or additional drugs in the pED identical to the drugs in the ambulance

'The five most common diagnose groups of patients transported to the $p E D$ 
suitable alternative for the initial assessment of some of these patients. However, previous studies also report that patients of all ages who were assessed to stay at the scene with or without triage protocols renew their contact with health care within $72 \mathrm{~h}$ in up to $19 \%$ of cases $[36,37]$. In this study, only $8.1 \%$ renewed their contact within $72 \mathrm{~h}$, with only two patients admitted to inpatient care. This indicates that, with few exceptions, the EMS nurse has the ability and knowledge to assess and allocate patients to other levels of care. Furthermore, another $31.7 \%$ of the patients who were transported to the pED were discharged by the pED nurse or by a physician without any intervention. Parental concern about their child's acuity and the feeling of security when transported by the EMS has been reported, even though it is not warranted [38], and this may sometimes explain why the EMS and the pED are contacted. A primary care centre located near the pED could be an alternative for this patient group. Another aspect is that more children who were non-conveyed were younger and, according to the EMS nurse, they could be managed by either self-care advice or treatment at the scene. On the other hand, the patients who were older were more predisposed to trauma and were therefore assessed as requiring $\mathrm{pED}$ resources due to for example a suspicion of a fracture.

Younger children were more frequently excluded from a triage colour, which could illustrate the difficulty of triaging an infant with VS according to the RETTS-p and the fact that this group were more likely to be non-conveyed. Patients with for example acute obstructive laryngitis where the primary RETTS-p assessment would indicate red or orange colour, were often thoroughly examined, treated and not conveyed. However, if triaged according to RETTS-p and only transported to the $\mathrm{pED}$ may have led to increased resource allocation in the pED.

This may indicate over-triage and the EMS nurse competence level, interventions on scene and the dynamic process of patient symptoms corresponding to the triage level in the EMS and later in the pED may play a role. Previous studies of the MTS similarly constructed as the RETTS-p have shown relatively high over-triage in the pED of $40-59 \%[10,11,39,40]$, where over-triage was mostly found in the lower triage categories [10]. There was a certain non-compliance to the RETTS protocol leading to missing variables. This may be a sign of mismatch between the protocol and the patient population. Thus the nurse overruled the protocol by clinical judgement, which sometimes may highlight the soundness of clinical management. This is why it may be wise to have a specialized nurse instead of a technician that may always follow an algorithm. The priorities of a triage system should be aimed at a high sensitivity to reduce under-triage for safety reasons. However, an excess of over-triage could have an impact on resource utilisation and pose a threat to safety for other patients. On the other hand, if the RETTS-p is already correctly applied in the pre-hospital setting, it is possible to downgrade the triage level for patients who are improved by an EMS nurse intervention. Furthermore, the mere definition of a triage tool is to determine who needs emergency care from a physician directly and who can wait, which does not include any decision on treatment and release. A computerised decision support tool may be introduced to guide the EMS nurse in the assessment, thus applying triage protocols when indicated for the utilisation of pED resources, which may increase compliance in the assessment process $[41,42]$.

The majority of the children were not completely triaged according to the RETTS-p. These patients did not receive a triage colour at all or were given a triage colour but with one or more VS missing. It could be suggested that an incomplete triage could jeopardise patient safety and thus omit significant abnormal vital signs. However, previous studies have shown that VS are only moderate predictors when assessing children in need of a trauma centre [43] and, furthermore, that there is a correlation between the degree of acuity level and the number of VS that were registered [44]. Even though measuring VS in the EMS care of injured and sick children has been emphasised to reduce under-triage $[45,46]$, implementing VS in all the patients in the MTS for paediatrics did not improve performance [47]. There are a number of reasons that may explain why not all VS according to the RETTS-p were measured in this study. They include a lower age, patients assessed as not being in need of VS measurement, short transport times and isolated and minor injuries in otherwise healthy children. This indicates that the RETTS-p is not feasible in the pre-hospital setting for all patients since it is not applied systematically. Other instruments such as the Patient assessment triangle (PAT) may be a viable alternative in the pre-hospital setting together with an A-E survey [48]. The PAT is a less complex tool and has shown to be reliable, accurate and easy to use at the initial assessment to evaluate the clinical status [49-51]. There is a concern that a relatively high proportion of the younger children in this study not had all their VS recorded. Serious infections such as sepsis and meningitis have a higher incidence with decreasing age [52]. A previous study reported that $15.4 \%$ of the children, presented at the pED with a suspected infection, were diagnosed with a serious infection which were significantly associated with deviation in VS [53]. Even though not all five VP were recorded according to RETTS-p in this study, it may be that when VS were reported by the EMS nurse it was 
clinically relevant in the current situation. Such a hypothesis is supported by the low frequency of renewed contact within $72 \mathrm{~h}$ and the fact that of those, only one patient was diagnosed with a potentially life-threatening diagnosis with no deviation in VS. Despite being implemented in the EMS as a triage instrument, the RETTS-p was never intended to assess whether or not patients could remain at the scene. The aim was to use it during transport to the pED and, among these patients, to estimate the time required until the patient needed to be examined by a physician based on the severity of the condition. This may reflect why so many patients who stayed at the scene lacked a triage colour. Furthermore, some patients did not receive a triage colour due to a severe critical condition (cardiac arrest, obstructed airway). In these cases, the activation of a medical/trauma team and the requirement of an examination by a physician immediately upon arrival was obvious. Five patients died within thirty days from EMS arrival in this study. Three patients were hospitalized and of those two patients died from a terminal disease.

\section{Strengths and limitations}

The major strength is that data were collected from a relatively large study cohort from a well-defined area in a systematic fashion, where data were prospectively reported. Major limitations were that data were collected from a single site in an urban setting with short transportation times which most likely influenced the results. The extrapolation of these data to other areas may therefore be problematic. Furthermore, due to the design of the study, data had to be collected from the patient records and important clinical parameters such as VS may have been measured but never recorded.

\section{Conclusions}

A representative study cohort of children below 16 years of age appeared to be safely assessed by the EMS nurse either to stay at the scene in one third of the cases or to be transported to the paediatric emergency department, regardless of whether the triage instrument was used to its full extent. However, one third of the patients were discharged from the pED with no interventions indicating an over-triage. The RETTS-p triage protocol may act as a tool to guide the EMS nurse in the assessment with application when feasible. The development of the RETTS-p triage protocol incorporated into a computerised decision support system, and the implementation of nurse-manned units specialising in children may further increase the potential to assess children and refer them to other levels of care, such as primary care. This would hopefully result in reduced overcrowding at the paediatric emergency department and more efficient resource utilisation with preserved patient care and safety.

\section{Abbreviations}

ALS: Advanced life support; ATS: Australasian triage scale; DMI: Dispatch medical index; EMS: Emergency medical services; ESI: Emergency severity index; ESS: Emergency signs and symptoms; GCS: Glasgow coma scale; ICD-10: International statistical classification of diseases and related health problems - tenth revision; MTS: Manchester triage system; PAT: Patient assessment triangle; pED: Paediatric emergency department; PedCTAS: Pediatric Canadian triage and acuity scale; RETTS-p: Rapid emergency triage and treatment system for paediatrics; VS: Vital signs

\section{Acknowledgements}

CM wishes to acknowledge LÖF, a nationwide Swedish insurance company which insures all publicly financed health-care providers, and the OLA Foundation (the foundation for prolonged and life-saving activities).

\section{Funding}

$C M$ received funding from LÖF and the OLA Foundation for the patient safety project of which this study is part.

\section{Availability of data and materials}

The datasets analysed during the current study are available from the corresponding author on reasonable request.

\section{Authors' contributions}

$\mathrm{CM}, \mathrm{CA}$ and $\mathrm{JH}$ developed the design and the research protocol. CM created the data forms and collected the data. TK supervised the statistical analysis. CM wrote the first draft. CA, JH and TK supervised and analysed the data and their interpretation. All the authors have participated in the final draft of the manuscript and have read and approved the final manuscript.

Ethics approval and consent to participate

All the procedures performed in this study were in accordance with the ethical standards of the Helsinki Declaration and the study was approved by the central ethical review board, Gothenburg, Sweden, Dnr: 970-15.

\section{Consent for publication}

Not applicable

Competing interests

The authors declare that they have no competing interests.

\section{Publisher's Note}

Springer Nature remains neutral with regard to jurisdictional claims in published maps and institutional affiliations.

\section{Author details}

${ }^{1}$ Department of Molecular and Clinical Medicine, Sahlgrenska Academy, University of Gothenburg, Gothenburg, Sweden. ${ }^{2}$ Pre Hospen-Centre for Prehospital Research, Faculty of Caring Science, Work Life and Social Welfare, University of Borås, Borås, Sweden. ${ }^{3}$ Health Metrics Unit, Sahlgrenska Academy, University of Gothenburg, Gothenburg, Sweden.

Received: 26 July 2018 Accepted: 12 October 2018

Published online: 19 October 2018

References

1. Travers DA, Waller AE, Bowling JM, Flowers D, Tintinalli J. Five-level triage system more effective than three-level in tertiary emergency department. J Emerg Nurs. 2002;28(5):395-400.

2. Fernandes CM, Tanabe P, Gilboy N, Johnson LA, McNair RS, Rosenau AM, Sawchuk P, Thompson DA, Travers DA, Bonalumi N, Suter RE. Five-level triage: a report from the ACEP/ENA five-level triage task force. J Emerg Nurs. 2005;31(1):39-50.

3. van Veen $M$, Moll HA. Reliability and validity of triage systems in paediatric emergency care. Scand J Trauma Resusc Emerg Med. 2009;17:38.

4. Ebrahimi M, Heydari A, Mazlom R, Mirhaghi A. The reliability of the Australasian triage scale: a meta-analysis. World J Emerg Med. 2015;6(2):94-9.

5. Allen AR, Spittal MJ, Nicolas C, Oakley E, Freed GL. Accuracy and interrater reliability of paediatric emergency department triage. Emerg Med Australas. 2015;27(5):447-52 
6. Ma W, Gafni A, Goldman RD. Correlation of the Canadian pediatric emergency triage and acuity scale to ED resource utilization. Am J Emerg Med. 2008;26(8):893-7.

7. Gravel J, Gouin S, Manzano S, Arsenault M, Amre D. Interrater agreement between nurses for the pediatric Canadian triage and acuity scale in a tertiary care center. Acad Emerg Med. 2008;15(12):1262-7.

8. Gravel J, Manzano S, Arsenault M. Validity of the Canadian paediatric triage and acuity scale in a tertiary care hospital. CJEM. 2009;11(1):23-8.

9. Green NA, Durani Y, Brecher D, DePiero A, Loiselle J, Attia M. Emergency severity index version 4: a valid and reliable tool in pediatric emergency department triage. Pediatr Emerg Care. 2012;28(8):753-7.

10. Roukema J, Steyerberg EW, van Meurs A, Ruige M, van der Lei J, Moll HA. Validity of the Manchester triage system in paediatric emergency care. Emerg Med J. 2006;23(12):906-10.

11. van Veen $M$, Steyerberg EW, Ruige M, van Meurs AH, Roukema J, van der Lei J, et al. Manchester triage system in paediatric emergency care: prospective observational study. BMJ. 2008;337:a1501.

12. Travers DA, Waller AE, Katznelson J, Agans R. Reliability and validity of the emergency severity index for pediatric triage. Acad Emerg Med. 2009;16(9): 843-9.

13. Baumann MR, Strout TD. Evaluation of the emergency severity index (version 3) triage algorithm in pediatric patients. Acad Emerg Med. 2005;12(3):219-24.

14. Al-Hindi AA, Al-Akhfash AA, Fareed AM, Alhusainan KS, Algasomy SF, Althowainy IR. Efficacy of implementation of a 5 scale pediatric triage and acuity scale in pediatric emergency. Saudi Arabia Saudi Med J. 2014;35(9):999-1004

15. Henning $B$, Lydersen $S$, Dollner $H$. A reliability study of the rapid emergency triage and treatment system for children. Scand J Trauma Resusc Emerg Med. 2016:24:19.

16. Westergren $H$, Ferm M, Haggstrom P. First evaluation of the paediatric version of the Swedish rapid emergency triage and treatment system shows good reliability. Acta Paediatr. 2014;103(3):305-8.

17. Cheung R, Ardolino A, Lawrence T, Bouamra O, Lecky F, Berry K, et al. The accuracy of existing prehospital triage tools for injured children in Englandan analysis using trauma registry data. Emerg Med J. 2013;30(6):476-9.

18. Lerner EB, Cushman JT, Drendel AL, Badawy M, Shah MN, Guse CE, et al. Effect of the 2011 revisions to the field triage guidelines on under- and over-triage rates for pediatric trauma patients. Prehosp Emerg Care. 2017; 21(4):456-60.

19. van Rein EAJ, Houwert RM, Gunning AC, Lichtveld RA, Leenen LPH, van Heijl M. Accuracy of prehospital triage protocols in selecting severely injured patients: a systematic review. J Trauma Acute Care Surg. 2017;83(2):328-39.

20. Voskens FJ, van Rein EAJ, van der Sluijs R, Houwert RM, Lichtveld RA, Verleisdonk EJ, et al. Accuracy of prehospital triage in selecting severely injured trauma patients. JAMA Surg. 2018;153(4):322-7.

21. Tsai LH, Huang CH, Su YC, Weng YM, Chaou CH, Li WC, et al. Comparison of prehospital triage and five-level triage system at the emergency department. Emerg Med J. 2017;34(11):720-5.

22. Seidel JS. Emergency medical services and the adolescent patient. J Adolesc Health. 1991;12(2):95-100.

23. Buschhorn HM, Strout TD, Sholl JM, Baumann MR. Emergency medical services triage using the emergency severity index: is it reliable and valid? J Emerg Nurs. 2013;39(5):e55-63.

24. Leeies M, Ffrench C, Strome T, Weldon E, Bullard M, Grierson R. Prehospital application of the Canadian triage and acuity scale by emergency medical services. CJEM. 2017;19(1):26-31.

25. Davies P, Maconochie I. The relationship between body temperature, heart rate and respiratory rate in children. Emerg Med J. 2009;26(9):641-3.

26. Khorram-Manesh A, Lennquist Montan K, Hedelin A, Kihlgren M, Ortenwall P. Prehospital triage, discrepancy in priority-setting between emergency medical dispatch Centre and ambulance crews. Eur J Trauma Emerg Surg. 2011;37(1):73-8.

27. Diggs LA, Sheth-Chandra M, De Leo G. Epidemiology of pediatric prehospital basic life support care in the United States. Prehosp Emerg Care. 2016;20(2):230-8.

28. Joyce SM, Brown DE, Nelson EA. Epidemiology of pediatric EMS practice: a multistate analysis. Prehosp Disaster Med. 1996;11(3):180-7.

29. Seid T, Ramaiah R, Grabinsky A. Pre-hospital care of pediatric patients with trauma. Int J Crit IIIn Inj Sci. 2012;2(3):114-20.

30. Tsai A, Kallsen G. Epidemiology of pediatric prehospital care. Ann Emerg Med. 1987;16(3):284-92.
31. Camasso-Richardson K, Wilde JA, Petrack EM. Medically unnecessary pediatric ambulance transports: a medical taxi service? Acad Emerg Med. 1997:4(12):1137-41.

32. Kost S, Arruda J. Appropriateness of ambulance transportation to a suburban pediatric emergency department. Prehosp Emerg Care. 1999;3(3):187-90.

33. Patterson PD, Baxley EG, Probst JC, Hussey JR, Moore CG. Medically unnecessary emergency medical services (EMS) transports among children ages 0 to 17 years. Matern Child Health J. 2006;10(6):527-36.

34. Richard J, Osmond MH, Nesbitt L, Stiell IG. Management and outcomes of pediatric patients transported by emergency medical services in a Canadian prehospital system. CJEM. 2006;8(1):6-12.

35. Dami F, Golay C, Pasquier M, Fuchs V, Carron PN, Hugli O. Prehospital triage accuracy in a criteria based dispatch Centre. BMC Emerg Med. 2015;15:32.

36. Magnusson C, Kallenius C, Knutsson S, Herlitz J, Axelsson C. Pre-hospital assessment by a single responder: the Swedish ambulance nurse in a new role: a pilot study. Int Emerg Nurs. 2016;26:32-7.

37. Ebben RHA, Vloet LCM, Speijers RF, Tonjes NW, Loef J, Pelgrim T, et al. A patient-safety and professional perspective on non-conveyance in ambulance care: a systematic review. Scand J Trauma Resusc Emerg Med. 2017;25(1):71.

38. Shah MN, Davis CO, Bauer C, Arnold J. Preferences for EMS transport and pediatric emergency department care. Prehosp Emerg Care. 2008;12(2):169-75.

39. van Veen $M$, Steyerberg EW, Van't Klooster M, Ruige M, van Meurs AH, van der Lei J, et al. The Manchester triage system: improvements for paediatric emergency care. Emerg Med J. 2012;29(8):654-9.

40. Seiger N, van Veen M, Steyerberg EW, van der Lei J, Moll HA. Accuracy of triage for children with chronic illness and infectious symptoms. Pediatrics. 2013;132(6):e1602-8

41. Andersson Hagiwara M, Suserud BO, Andersson-Gare B, Sjoqvist B, Henricson $M$, Jonsson $A$. The effect of a computerised decision support system (CDSS) on compliance with the prehospital assessment process: results of an interrupted time-series study. BMC Med Inform Decis Mak. 2014;14:70.

42. Hagiwara MA, Sjoqvist BA, Lundberg L, Suserud BO, Henricson M, Jonsson A. Decision support system in prehospital care: a randomized controlled simulation study. Am J Emerg Med. 2013;31(1):145-53.

43. Lerner EB, Drendel AL, Cushman JT, Badawy M, Shah MN, Guse CE, et al. Ability of the physiologic criteria of the field triage guidelines to identify children who need the resources of a trauma center. Prehosp Emerg Care. 2017;21(2):180-4.

44. Gravel J, Opatrny L, Gouin S. High rate of missing vital signs data at triage in a paediatric emergency department. Paediatr Child Health. 2006;11(4): 211-5.

45. Hewes $H$, Hunsaker $S$, Christensen M, Whitney J, Dalrymple T, Taillac P. Documentation of pediatric vital signs by EMS providers over time. J Pediatr Surg. 2016;51(2):329-32.

46. Seiger $N$, van Veen $M$, Steyerberg EW, Ruige $M$, van Meurs $A H$, Moll HA. Undertriage in the Manchester triage system: an assessment of severity and options for improvement. Arch Dis Child. 2011;96(7):653-7.

47. Seiger $N$, van Veen M, Almeida H, Steyerberg EW, van Meurs AH, Carneiro R, et al. Improving the Manchester triage system for pediatric emergency care: an international multicenter study. PLoS One. 2014;9(1):e83267.

48. Fuchs S, Terry M, Adelgais K, Bokholdt M, Brice J, Brown KM, et al. Definitions and assessment approaches for emergency medical services for children. Pediatrics. 2016;138(6):e20161073.

49. Dieckmann RA, Brownstein D, Gausche-Hill M. The pediatric assessment triangle: a novel approach for the rapid evaluation of children. Pediatr Emerg Care. 2010;26(4):312-5.

50. Gausche-Hill M, Eckstein M, Horeczko T, McGrath N, Kurobe A, Ullum L, et al. Paramedics accurately apply the pediatric assessment triangle to drive management. Prehosp Emerg Care. 2014;18(4):520-30.

51. Horeczko T, Enriquez B, McGrath NE, Gausche-Hill M, Lewis RJ. The pediatric assessment triangle: accuracy of its application by nurses in the triage of children. J Emerg Nurs. 2013;39(2):182-9.

52. Van den Bruel A, Bartholomeeusen S, Aertgeerts B, Truyers C, Buntinx F. Serious infections in children: an incidence study in family practice. BMC Fam Pract. 2006;7:23.

53. Thompson M, Coad N, Harnden A, Mayon-White R, Perera R, Mant D. How well do vital signs identify children with serious infections in paediatric emergency care? Arch Dis Child. 2009;94(11):888-93. 